\title{
A Semi Analytic Approach to Coupled Boundary Value Problem
}

\author{
Nityanand P. Pai, Nagaraj N. Katagi \\ Department of Mathematics, Manipal Institute of Technology, Manipal University, Manipal, India \\ Email: nppaimit@yahoo.co.in, nnkatagi@yahoo.com
}

Received 1 July 2014; revised 2 August 2014; accepted 15 August 2014

Copyright (C) 2014 by authors and Scientific Research Publishing Inc.

This work is licensed under the Creative Commons Attribution International License (CC BY). http://creativecommons.org/licenses/by/4.0/

c) (i) Open Access

\begin{abstract}
The present problem is considered as a coupled boundary value problem and is analyzed using a semi analytic method. A series method is used to obtain the solution and region of validity is extended by suitable techniques. In this case of series solution the results obtained are better than pure numerical findings up to moderately large Reynolds numbers. The variation of physical parameters is discussed in detail.
\end{abstract}

\section{Keywords}

\section{Suction, Coupled Equations, Analytic Continuation, Physical Parameters, Reynolds Numbers}

\section{Introduction}

The flow between porous discs has been studied by several authors. As in the case of porous pipes and porous channels, the governing equation reduces to a set of nonlinear ordinary differential equations. This problem was first studied by Batchelor [1] who generalized the solution of Von Karman [2]. Further this problem was analyzed by Stewartson [3] who obtained perturbation solution for the small Reynolds number. Flow between a rotating and a stationary disc has been studied by Phan-Thein and Bush [4]. The problem of suction of viscous in incompressible fluid through a rotating porous disc onto a rotating co-axial disc was studied by C. Y. Wang [5]. Here we view this problem as a coupled nonlinear boundary value problem.

In the present paper we have used semianalytical numerical technique to understand the effect of suction represented by a pair of nonlinear differential equations. For simple geometrics the semianalytical numerical methods proposed here provide better results. Van Dyke [6] and his associates have successfully used these series methods in unveiling important features of various types of fluid flows. Bujurke and Pai [7] has discussed similar geometrical problem with injection at the discs.

Recently in the analysis of flow in pipe, Pai and Katagi [8] have used series analysis satisfactorily. The phys- 
ical problem considered in this paper is of great importance in Fluid Dynamics. The present study is a possible extension work of Wang [5]. The problem is solved using a special type of series. The series so generated has limited utility due to presence of a singularity and its region of validity is extended by analytic continuation.

\section{Mathematical Formulation}

As shown in the Figure 1 we denote the spacing between the discs by $z= \pm L$ and rotating with the same angular velocity $\Omega$. Fluid is withdrawn from both discs with velocity $W$. We shall assume the gap with $2 L$ is small compared to diameter of the discs. So that end effects can be neglected. The flow field is symmetric about the $z=0$.

The governing equations are

$$
\begin{gathered}
u u_{r}+w u_{z}-\frac{v^{2}}{r}=-\frac{P_{r}}{\rho}+v\left(\nabla^{2} u-\frac{u}{r^{2}}\right) \\
u v_{r}+w v_{z}+\frac{u v}{r}=v\left[\nabla^{2} v-\frac{v}{r^{2}}\right] \\
u w_{r}+w w_{z}=-\frac{P_{z}}{\rho}+v \nabla^{2} W \\
(r u)_{r}+r w_{z}=0
\end{gathered}
$$

where $\nabla^{2}=\frac{\partial^{2}}{\partial r^{2}}+\frac{1}{r} \frac{\partial}{\partial r}+\frac{\partial^{2}}{\partial z^{2}}$, and $u, v, w$ are velocity components. The boundary conditions are at $z= \pm L$, $u=0, v=r \Omega, w= \pm W$.

For similarity transformation we consider Wang [5]

$$
\begin{aligned}
& u=r f^{\prime}(\eta) \frac{W}{L}, \\
& v=r g(\eta) \frac{W}{L}, \\
& w=-2 f(\eta) W, \\
& p=-\rho r^{2} A \frac{W^{2}}{\left(2 L^{2}\right)}+v P(\eta),
\end{aligned}
$$

where $\eta=\frac{z}{d}$ and $A$ is constant. Now the equations take the form

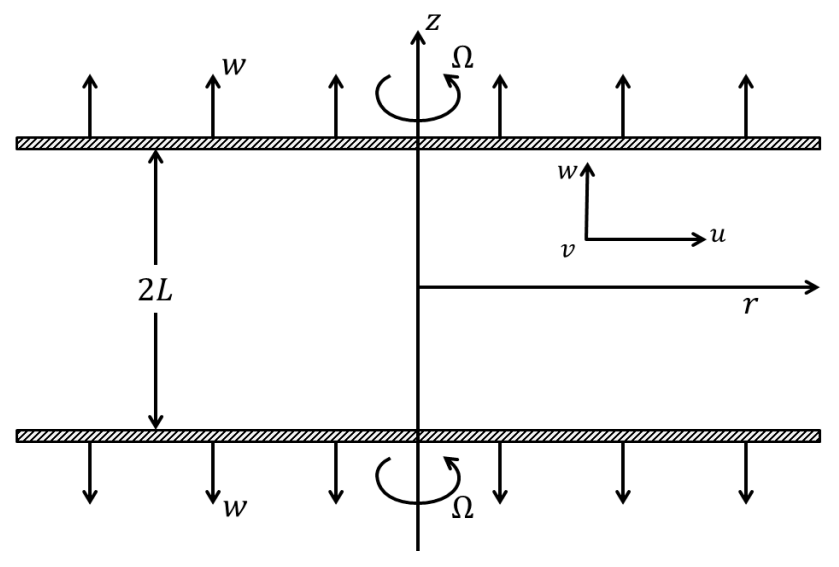

Figure 1. The co-ordinate system. 


$$
\left(f^{\prime}\right)^{2}-2 f f^{\prime \prime}-g^{2}=A+\frac{1}{R} f^{\prime \prime \prime}
$$

or

$$
\begin{gathered}
-2 R\left(f f^{\prime \prime \prime}+g g^{\prime}\right)=f^{i v} \\
2 R\left(f^{\prime} g-f g^{\prime}\right)=g^{\prime \prime} \\
P(\eta)=\left(-2 f^{2} W^{2}-2 v W \frac{f^{\prime}}{L}\right)+B .
\end{gathered}
$$

Here $R=\frac{W d}{v}$ is Reynolds number. The boundary conditions are

$$
\begin{gathered}
f^{\prime}(1)=f(0)=f^{\prime \prime}(0)=0, \quad f(1)=-\frac{1}{2}, \\
g^{\prime}(0)=0, \quad g(1)=\frac{\Omega L}{W}=\beta .
\end{gathered}
$$

\section{Method of Solution}

We assume the solution of (1.6) and (1.7) in the forms

$$
\begin{gathered}
f(\eta)=f_{0}(\eta)+\sum_{n=1}^{\infty} R^{n} f_{n}(\eta) \\
g(\eta)=g_{0}(\eta)+\sum_{n=1}^{\infty} R^{n} g_{n}(\eta) .
\end{gathered}
$$

Substituting (3.1), (3.2) in (1.6) and (1.7) and comparing powers of $R$ one can get

$$
\begin{gathered}
f_{n}^{i v}=-2 \sum_{n=1}^{\infty} f_{r} f_{n-1-r}^{\prime \prime \prime}-g_{r} g_{n-1-r}^{\prime} \\
g_{n}^{\prime \prime}=2 \sum_{n=1}^{\infty}\left(f_{r}^{\prime} f_{n-1-r}-f_{r} g_{n-1-r}^{\prime}\right) \quad n=0,1,2 \cdots .
\end{gathered}
$$

The relevant boundary conditions are

$$
\begin{gathered}
f^{\prime}(1)=0, \quad f_{0}(0)=f_{0}^{\prime \prime}(0)=0, \quad f_{0}(1)=-\frac{1}{2}, \\
g_{0}^{\prime}(0)=0, \quad g(1)=\beta, \\
f_{n}^{\prime}(1)=0, \quad f_{n}(0)=0, \quad f_{n}^{\prime \prime}(0)=0, \quad f_{n}(1)=0, \\
g_{n}^{\prime}(1)=0, g_{n}(1)=0, \quad n=1,2,3 \cdots
\end{gathered}
$$

The solutions are

$$
\begin{gathered}
f_{0}=\frac{1}{4}\left(\eta^{3}-3 \eta\right), \quad g_{0}=\beta, \\
f_{1}=\frac{1}{1120}\left(-\eta^{7}+21 \eta^{5}-39 \eta^{2}+19 \eta\right), \\
g_{1}=\frac{\beta}{8}\left(\eta^{4}-6 \eta^{2}+5\right), \\
f_{2}=\frac{1}{560}\left\{\frac{-3}{440} \eta^{11}+\frac{1}{6} \eta^{9}-\frac{177}{140} \eta^{7}+\frac{17}{10} \eta^{5}-\frac{443}{1848} \eta^{3}-\frac{137}{385} \eta\right\}-\beta^{2}\left(\frac{1}{840} \eta^{7}-\frac{1}{40} \eta^{5}+\frac{13}{280} \eta^{3}-\frac{19}{840} \eta\right),
\end{gathered}
$$




$$
g_{2}=\beta\left(\frac{-3}{2240} \eta^{8}+\frac{1}{80} \eta^{6}-\frac{111}{3360} \eta^{4}-\frac{253}{560} \eta^{2}+\frac{3183}{6720}\right) .
$$

The slow convergence of series (3.1), (3.2) requires large number of terms for obtaining the approximate sum. For higher order approximations we proceed by the following scheme.

We propose a systematic scheme for this purpose, we consider $f_{n}$ and $g_{n}$ to be of the forms

$$
\begin{aligned}
& f_{n}(\eta)=\sum_{k=2}^{4 n+1} C_{n, k} \eta^{k}(1-\eta)^{2} \\
& g_{n}(\eta)=\sum_{n=1}^{4 n} D_{n, k} \eta^{k}(1-\eta) .
\end{aligned}
$$

We substitute (3.5), (3.6) in (3.3), (3.4) respectively and equate various powers of $\eta$ on both sides to get

$$
\begin{aligned}
C_{n(k)}= & 2 C_{n(k+1)}-C_{n(k+2)}+\frac{1}{(k+2)(K+1)(k)(k-1)} \times\left\{\sum_{i=1}^{6} C_{(n-1)(k+2-i)} P_{i(k+2-i)}+\sum_{i=1}^{3} D_{(n-1)(k-i)} Q_{i(k-i)}\right. \\
+ & \left.\sum_{r=1}^{n-2}\left[\sum_{i=0}^{4} \sum_{j=1}^{n k+i} C_{r(\ell+1-n k-i)} C_{(m-1)(t-2)} P_{7+i}(t-2)+\sum_{r=0}^{2} \sum_{j=1}^{n k+i} D_{r(\ell-n k-i)(t-3)} D_{(m-1)(t-3)} Q_{(4+i)}(t-3)\right]\right\}, \\
D_{n(k)}= & D_{n(k+1)}-\frac{1}{k(k+1)}\left[\sum_{i=1}^{5} D_{(n-1)(k+1-i)} T_{i}(k+1-i)+\sum_{i=1}^{4} C_{(n-1)(k+1-i)} T_{i}^{\prime}(k+1-i)\right. \\
& \left.+\sum_{r=1}^{n-2} \sum_{i=0}^{3} \sum_{j=1}^{n k+i} C_{(\ell+1-n k-i)} D_{(m-1)(t-3)} \Gamma_{6+i}(\ell+1-n k-i, t-3)\right],
\end{aligned}
$$

where $m=n-4, l=4 r+j, t=4 m-j, n k=4 n-2-k, P_{i}, Q_{i}, T_{i}$ 's are functions of $k$ and $k_{1}$.

For the radial velocity profile,

$$
f^{\prime}(\eta)=\frac{1}{4}\left(3 \eta^{2}-3\right)+\sum_{n=1}^{\infty} R^{n} \sum_{k=2}^{4 n+1} C_{n(k)}-\left(k \eta^{k-1}-2(k+1) \eta^{k}+(k+2) \eta^{k+1}\right) .
$$

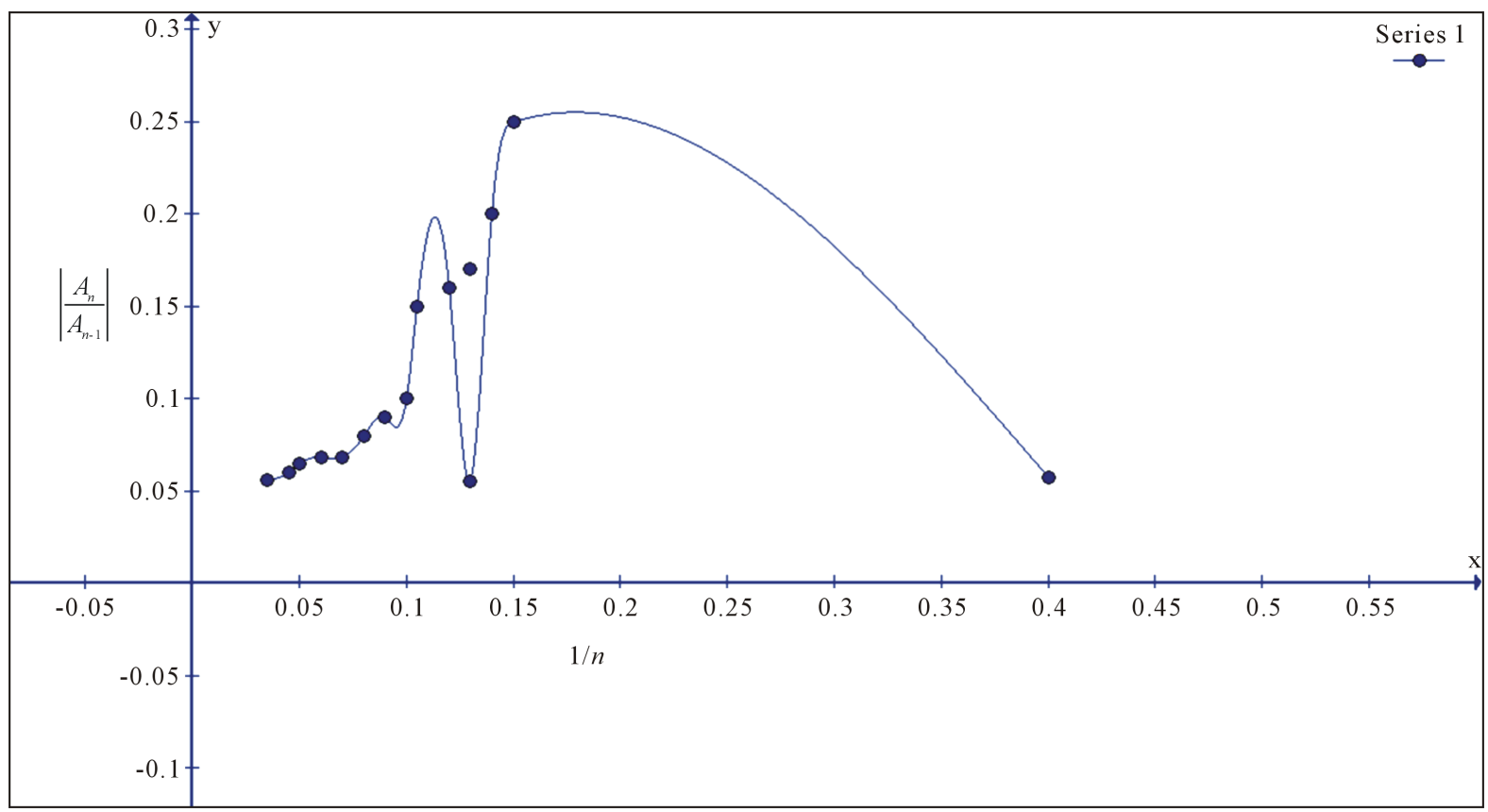

Figure 2. Domb sykes plot. 
Table 1. Comparison of values for $f^{\prime}(0)$.

\begin{tabular}{|c|c|c|c|}
\hline$R$ & $\beta$ & $f^{\prime}(0)$ (numerical) & Series method \\
\hline 40.812 & 3.00 & -------- & -0.34121 \\
\hline 20.345 & 3.6648 & -0.54398 & -0.54398 \\
\hline 0.13151 & 0.68501 & -0.74150 & -0.74150 \\
\hline-39.0691 & 0.98224 & -0.6550 & -0.65511 \\
\hline-60.000 & 0.98204 & -------- & -0.41511 \\
\hline
\end{tabular}

Table 2. Comparison of values for $f^{\prime \prime \prime}(0)$.

\begin{tabular}{|c|c|c|c|}
\hline$R$ & $\beta$ & $f^{\prime \prime \prime}(0) \quad$ (numerical) & Series method \\
\hline 40.812 & 3.00 & -------- & 0.0000 \\
\hline 20.345 & 3.6648 & 0.0069 & 0.00688 \\
\hline 8.5679 & 0.000 & 0.24870 & 0.24871 \\
\hline 0.13151 & 0.68501 & 1.37862 & 1.37862 \\
\hline-4.39563 & 0.43440 & 2.24572 & 2.24571 \\
\hline-39.0691 & 0.98224 & 0.00156 & 0.00156 \\
\hline-60.000 & 0.98204 & -------- & 0.00000 \\
\hline
\end{tabular}

And we have

$$
f^{\prime \prime \prime}(\eta)=\frac{3}{2}+\sum_{m=1}^{\infty} R^{n} \sum_{k=2}^{4 n+1} C_{n(k)}\left(k(k-1)(k-2) \eta^{k-2}-2(k+1)(k)(k-1) \eta^{k-1}+(k+2)(k+1) k \eta^{k}\right)
$$

\section{Conclusions}

A new type of series is presented for studying the problems of flow between the two discs using recurrences (3.7) and (3.8). In this process we generate universal coefficients $C_{n(k)}, \eta=1, \cdots, 30, k=4 n+1$.

These coefficients in turn give universal coefficients for $f_{n}(\eta)(n=1,2, \cdots, 30)$. The series (3.9) and (3.10) give $f^{\prime}(0)$ and $f^{\prime \prime \prime}(0)$ respectively using Domb-sykes plot (Figure 2), and we locate the position of singularities for $\beta=0$ at 17.89. The series (3.9) and (3.10) are summed using Pade-approximants. Earlier numerical results were from $R=20$ to -39 . We are able to go upto $R=40$ to -60 using Pade-approximants. The results are in close agreement with numerical findings of Wang [5] which are shown in the Table 1 and Table 2.

\section{References}

[1] Batchelor, G.K. (1951) Note on Class of Solution of Navier-Stockes Equations Representing Symmetric Flow. Quarterly Journal of Mechanics Applied Mathematics, 4, 29-35. http://dx.doi.org/10.1093/qjmam/4.1.29

[2] Van Karman, T. (1921) Laminare and Turbulent Reibung. Zeitschrift für Angewandte Mathematik und Mechanik, 1, 233-241. http://dx.doi.org/10.1002/zamm.19210010401

[3] Stewertson, K. (1953) On the Flow between Two Rotating Discs. Proceedings of the Cambridge Philosophical Society, 49, 233-340.

[4] Phan Thein, N. and Bush, M.B. (1984) On the Steady Flow of a Newtonian Fluid between the Parallel Disk. Zeitschrift für Angewandte Mathematik und Physik, 35, 912-919. http://dx.doi.org/10.1007/BF00945453

[5] Wang, C.Y. (1986) Symmetric Viscous Flow between Two Rotating Porous Disc. Quarterly of Applied Mathematics, 4, 29-37.

[6] Van Dyke, M. (1974) Analysis and Improvement of Perturbation Series. Quarterly Journal of Mechanics Applied Mathematics, 27, 423-456. http://dx.doi.org/10.1093/qjmam/27.4.423 
[7] Bujurke, N.M. and Pai, N.P. (1995) Computer Extended Series Solution to Viscous Flow between Rotating Discs. Proceedings of the Indian Academy of Sciences (Mathematical Sciences), 105, 353-369. http://dx.doi.org/10.1007/BF02837202

[8] Pai, N.P. and Katagi, N.N. (2013) Semi Numerical Solution for a Boundary Value Problems. American Journal of Computational Mathematics, 3, 43-47. http://dx.doi.org/10.4236/ajcm.2013.31006 
Scientific Research Publishing (SCIRP) is one of the largest Open Access journal publishers. It is currently publishing more than 200 open access, online, peer-reviewed journals covering a wide range of academic disciplines. SCIRP serves the worldwide academic communities and contributes to the progress and application of science with its publication.

Other selected journals from SCIRP are listed as below. Submit your manuscript to us via either submit@scirp.org or Online Submission Portal.
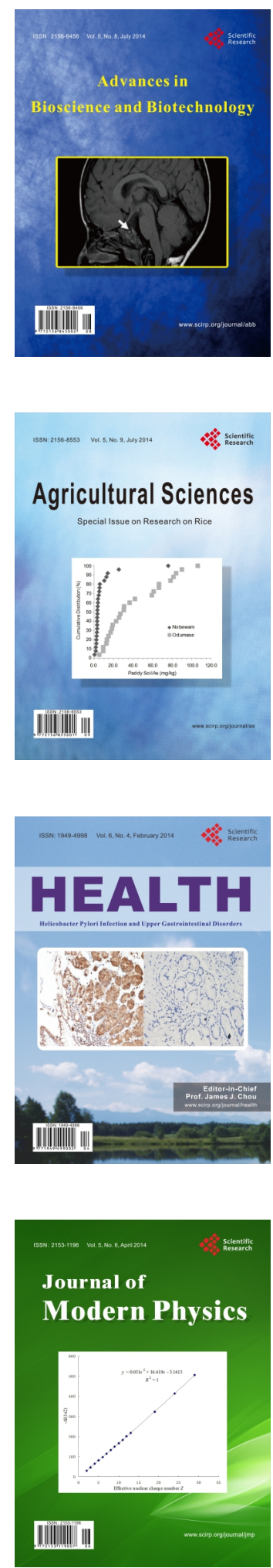
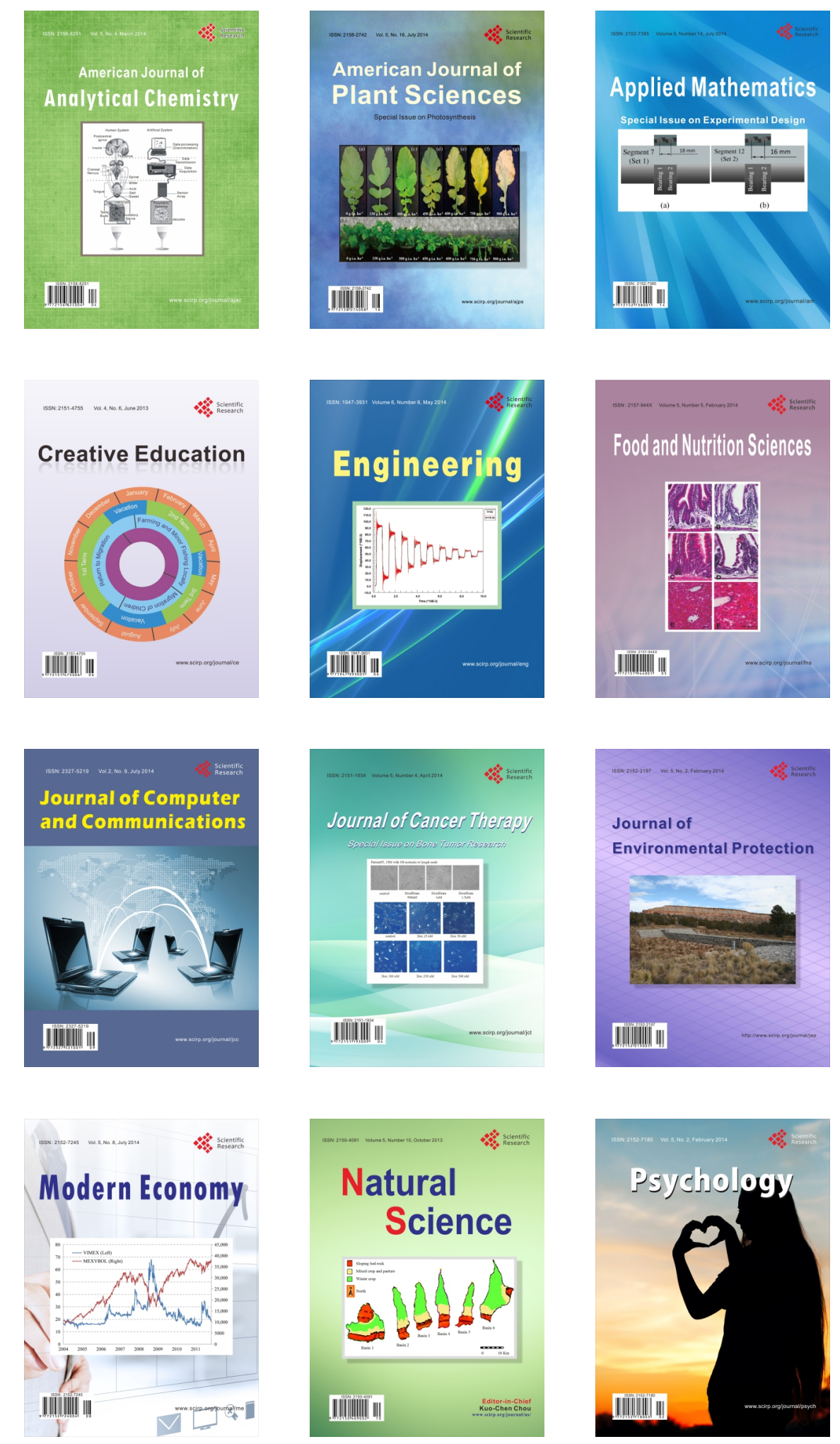